\title{
Análisis de redes empresariales y puertas giratorias en México: Cartografía de una clase dominante público-privada
}

\section{Analysis of Business Elite Networks and Revolving Doors in Mexico: Cartography of a Public-Private Ruling Class}

\section{Jaime Aragón Falomir y Julián Cárdenas}

\author{
Jaime Aragón Falomir es becario posdoctoral del \\ Consejo Nacional de Investigaciones Científicas y \\ Técnicas de Argentina y Profesor en la Université des \\ Antilles, Francia. \\ E-mail: jaime.aragonf@gmail.com
}

\section{resumen}

La presente investigación mapea las redes de las élites empresariales y políticas en México para comprender la organización social y la capacidad de influencia de las élites mexicanas, y abrir vías de investigación sobre las élites de poder en México. En primer lugar, se realiza un estudio de la cohesión interna de las élites empresariales mediante un análisis de redes de interlocking directorates y enlaces de propiedad. En segundo lugar, se examinan varios casos de puertas giratorias mediante un análisis prosopográfico de biografías y trayectorias. Los resultados revelan que las élites empresariales mexicanas están cohesionadas y que existe un grupo de individuos que fluctúan entre el sector público y el privado. Además, la combinación de ambos análisis muestra que las empresas o grupos económicos con acceso al Estado vía puertas giratorias son empresas bien conectadas en la red de interlocking directorates. Estos resultados sugieren la emergencia de una clase dominante públicoprivada en México.

\section{palabras clave}

élites empresariales / élites políticas / puertas giratorias / redes de élites / México
Julián Cárdenas es profesor en la Universidad de Valencia, España.

E-mail: julian.cardenas@onlinebschool.com

\section{summary}

This research maps the business and political elite networks in Mexico to understand the social organization and influence capacity of Mexican elites, and to open up avenues of research on power elites in Mexico. First, a study of the internal cohesion of the business elites is carried out through an analysis of networks of interlocking directorates and ownership ties. Secondly, we examine several cases of revolving doors through a prosopographic analysis of biographies and trajectories. The results reveal that the Mexican business elites are cohesive and that a group of individuals fluctuate between the public and private sectors. In addition, the combination of both analyses shows that corporations or business groups with access to the State via revolving doors are well-connected firms in the network of interlocking directorates. Findings suggest the emergence of a publicprivate ruling class in Mexico.

\section{keywords}

business élites / political elites / revolving doors / elite networks / Mexico 


\section{Introducción}

Desde que el expresidente Carlos Salinas de Gortari toma el poder en México (1988), hasta la contemporánea nominación de José A. Meade como candidato presidencial (2018) del Partido Revolucionario Institucional (PRI), es posible encontrar un hilo conductor. Durante estos treinta años, una red de actores empresariales ha mantenido un acceso inquebrantable al poder político y una inédita continuidad en sectores económico-financieros. Esta es la razón por la cual Aitken declara que "Salinas debe ser reconocido como el padre del actual sistema económico y político de México" (2008: 459). Para entender tanto las estructuras de poder como el entorno económico, son necesarios estudios sobre las élites y más concretamente sobre cómo se enredan o relacionan las élites entre sí (Cárdenas, 2016a).

A pesar de que existen diversos estudios sobre las élites en México (Hernández Rodríguez, 1998; Rousseau, 1999; Camp, 2002, 2010, 2012; Salas-Porras y Luna Ledesma, 2012), continúan emergiendo debates acerca de la organización de las élites, y sobre el vínculo entre las élites empresariales y políticas. Con respecto a la organización de las élites mexicanas, Salas-Porras (2006) mostró un debilitamiento de las redes empresariales en los años 2000, mientras que Cárdenas (2016b) evidenció una alta cohesión interna a través de los consejos de administración. Por otro lado, el paso de políticos al sector privado y de empresarios a la política (fenómeno conocido como puertas giratorias o pantouflage) es destacado por algunos autores (Rouban, 2010; Tirado, 2012; Durand, 2016; Castellani, 2018), pero minimizado por otros (Babb, 2003; Rousseau, 1999; Mendieta y Schmidt, 2005), incluso algunos indican que es "una discusión ya superada" (Hernández y Pansters, 2012: 761). Estos debates provocan dos inquietudes. ¿Existe una élite o clase dominante público-privada en México? Si así fuera, ¿está basada en una organización de redes cohesionada? Para contribuir a estos debates e inquietudes, se realiza un análisis que abarque tanto a las élites políticas como empresariales, que esté basado en amplios datos empíricos y no solo en evidencia anecdótica, y que emplee una aproximación de redes para visualizar la posible formación de una élite público-privada en México desde el gobierno de Salinas (1998) hasta la llegada de un gobierno de izquierdas con Andrés Manuel López Obrador (2018).

Específicamente, la presente investigación mapea las relaciones de las élites mexicanas para comprender su organización y su capacidad de influencia. Las relaciones de las élites son analizadas en dos fases. En primer lugar, se realiza un estudio minucioso de las redes entre las élites empresariales en México mediante un análisis de interlocking directorates y enlaces de propiedad entre las grandes empresas, para así averiguar el nivel cohesión o dispersión del poder económico. Este análisis nos permite entender la estructura que se beneficia y también sustenta a los actores que se mueven entre el sector público y el privado.

En segundo lugar, se indaga y revela cómo y cuándo empezó a haber flujos de y hacia el sector político y empresarial mediante un seguimiento prosopográfico (Ferrari, 2010). Para eso, se profundiza sobre algunos casos de actores que pasaron del sector público al privado. De esta forma, se identifican los conectores de redes 
político-empresariales revelando las puertas giratorias (pantouflage), es decir, "el hecho que un funcionario deje su puesto público para trabajar en el sector privado, beneficiándose de las distintas disposiciones que le permite la movilidad como funcionario" (Rouban, 2010: 23).

Los hallazgos de esta investigación permiten comprender las estructuras latentes en las que se apoya la influencia del sector empresarial en la política y, en consecuencia, se favorecen discusiones sobre la posible captura del Estado en México. Aunque han crecido notablemente los estudios que analizan las élites para entender la captura del Estado (Durand, 2016; Serna y Botinelli, 2018), son mínimos los que han incorporado el análisis de las redes de las élites (Cárdenas y RoblesRivera, 2018). Por lo tanto, el presente artículo permite abrir líneas de investigación en estos campos de estudio y generar un estímulo para futuras pesquisas.

\section{Las élites en México}

Scott (2008) propone que las élites son aquellos grupos sociales que tienen y ejercen el poder, el cual es definido como el efecto causal de un actor sobre el comportamiento de otros. Aquellos que tienen más relaciones de poder tienen la autorización de disciplinar a otros (influencia correctiva e influencia persuasiva) ejerciendo entonces la dominación. Solo a estos grupos se les puede llamar élites (Scott, 2008: 29-35). No obstante, para ejercer la dominación, Boltanski (2009) indica que es necesario que los actores estén dispersos en un espacio, que ejerzan actividades diferentes y puestos diversos, con poderes desiguales. Pertenecer a la clase dominante es estar convencido de que puede apoyarse en redes deslocalizadas y dispositivos sociales complejos (2009: 213-214). Por tanto, las élites son estudiadas a partir de sus relaciones internas, pero con impacto externo más allá de su propio sector o ámbito.

El análisis de redes es la aproximación teórica y metodológica que relanzó los estudios de las élites a partir de la década de 1990. Aunque antes se habían incorporado nociones de redes como la integración o cohesión para investigar a las élites de poder (Mills, 1956), es a partir de la emergencia del análisis de redes y los programas informáticos asociados que se pudo recolectar grandes cantidades de datos sobre los vínculos sociales y los espacios de pertenencia de las élites. Tras la proliferación de investigaciones sobre redes entre las élites empresariales durante los años 2000 y 2010 para retratar la organización del poder económico (Rodríguez, Cárdenas y Oltra, 2006; Rodríguez, 2003), surgieron estudios empíricos sobre cómo esas mismas redes permiten entender la influencia política de los grandes empresarios (Mizruchi, 2016). Por ello, resulta clave analizar la organización social de las élites, tanto de los grandes empresarios entre sí, como de los vínculos con el sector público, ya que permiten cartografiar los vínculos públicoprivados e identificar las estructuras que los sustentan.

\section{La organización social de las élites empresariales}

De las diversas formas en que se "enredan" las élites empresariales (directivos y accionistas de las grandes empresas), los interlocking directorates y los enlaces 
de propiedad son mecanismos que configuran estructuras estables y muestran las relaciones internas del poder económico. Los interlocking directorates son relaciones entre empresas creadas por los directivos al pertenecer a varios consejos de administración. Los enlaces de propiedad son relaciones entre grandes accionistas por tener inversiones conjuntas en las grandes empresas. El análisis de los interlocking directorates y los enlaces de propiedad muestran las redes de las élites empresariales permitiendo conocer su grado de cohesión o dispersión. La influencia de las élites empresariales mexicanas en la política ha sido magnánima, por lo que el análisis de cómo están organizadas las élites empresariales internamente revela las formas que potencian lo que se ha dado en denominar el poder empresarial (Fuchs, 2007) o captura del Estado (Durand, 2016). Para descubrir y analizar cómo están organizadas las élites empresariales en México, se examina la cohesión o unión de las élites empresariales en dos sectores: redes de interlocking directorates y redes de propiedad. De esta manera, se comprenden las estructuras relacionales en las que se sustenta la influencia del sector empresarial en la política.

Los interlocking directorates (enlaces entre empresas por compartir directivos) son un indicador relacional para examinar cómo las élites empresariales están interconectadas y descubrir las redes que configuran. La literatura sobre redes empresariales ha destacado que los interlocking directorates son fuentes de información y control, por lo que las redes empresariales cohesionadas facilitan la coordinación y la acción colectiva (Useem, 1984). Los enlaces de propiedad (también conocidos como interlocking ownership) son otro indicador relacional para medir el grado de unión o desunión entre las élites propietarias. Cuando en una misma gran empresa hay más de un propietario significativo (que controla más del 5\% del capital accionarial), se crea un enlace entre estos grandes accionistas.

\section{La incursión politica de las élites empresariales en México}

Aunque durante una parte importante del siglo XX la participación del sector empresarial en política fue timorata, esa "simbiosis con capacidad para perdurar" (Alba Vega, 2006: 124) se modificó drásticamente a partir del final de la década de 1980. Se observaron tres vías de implicación de las élites empresariales en la política: I) la búsqueda de puestos de elección generalmente a través del Partido Acción Nacional (PAN) con el que el sector empresarial comparte posiciones ideológicas (Ortiz Gallegos, 2011; Loaeza, 2010; Luna Ledesma y Tirado, 1986); II) el acercamiento hacia el gobierno del PRI, liderado por C. Salinas (1988-1994) a través de la privatización de empresas públicas que beneficiaron a una parte del empresariado (Babb, 2003; Alba Vega, 1996); y III) estrategias de cabildeo o lobbying a través del Consejo Coordinador Empresarial (CCE) o el Consejo Mexicano de Hombres de Negocios (Alba Vega, 2006: 147).

En 1989, las listas de multimillonarios de Forbes solo incluían un mexicano, el empresario cervecero de Monterrey (hoy FEMSA) Eugenio Garza Sada. No obstante, a partir de ese año, el número de empresarios se multiplicó. Por ejemplo, Carlos Slim Helú pasó de no estar en el ranking antes de 2005 a ocupar los primeros puestos durante más de una década. Este incremento de grandes fortunas 
en México implicó que los empresarios se independizaron económicamente del Estado (Aguayo, 2007: 251), pudiendo postularse como oposición política, obteniendo la presidencia en el año 2000 con Vicente Fox, fenómeno que se extendió posteriormente en la región (Serna, 2018).

Por otro lado, en 1987, el PRI, partido gobernante, sufre la escisión más grande de su historia. Los actores inconformes compitieron contra su antiguo partido provocando una de las elecciones más polémicas y fraudulentas de México, que finaliza con la toma de posesión de C. Salinas en 1988. Labastida y Camou señalan que en ese momento "los políticos profesionales son desplazados por los tecnócratas como antes aquéllos desplazaron a los militares", lo cual evidencia un "triunfo de la tecnocracia sobre los políticos tradicionales" (2001: 205-2010). Esto apresuró el proceso de apertura democrática-electoral, ya que el PRI estuvo "obligado" a concertar y ceder algunas gubernaturas (Baja California en 1989 y Chihuahua en 1991) que concluirán con la llamada transición en el año 2000 (Woldenberg, 2012).

Por lo tanto, en los años 1980 y 1990, observamos la modificación de distintos paradigmas tanto del sector empresarial como del gubernamental, que conlleva un reordenamiento interno y externo, afectando la vida político-económica del país. El sector empresarial se vinculó tanto con el PAN (cohabitación con panistas de antaño) como con el PRI (a través de las privatizaciones, conviviendo con priistas tradicionales). Esto se dio a la par del inicio de un proceso de democratización electoral que supone un reto para las élites de poder en México.

Smith (1976) y Camp $(1995,2002)$ auguraban una modificación en los modos de reclutamiento de las élites mexicanas. Si bien las élites tradicionales sufrieron una ruptura en la cúpula desde 1988, considerada como "una revolución silenciosa" (Rousseau, 1999), hubo también un discreto "flujo de apertura actoral" que implicó el acceso de tecnócratas-políticos (Technopols) dentro de la arena política (Domínguez, 1997). Aunque este fenómeno se reprodujo en gran parte de América Latina durante la década de 1990 (Dezalay y Garth, 2002), la particularidad mexicana pareciera haberse dado antes de su apertura democrático-electoral del año 2000. ¿De dónde fueron reclutados estos nuevos tecnócratas-políticos? ¿Qué vínculos tenían los tecnócratas políticos con las élites empresariales? ¿Eran miembros de las élites empresariales? ¿Emergió una élite o clase dominante público-privada? El estudio de la organización de las élites empresariales y de una serie de casos emblemáticos de puertas giratorias (o pantouflage) permite responder estas incógnitas y cartografiar a las élites en México, así como abrir la caja negra de las élites de poder en México.

\section{Métodos y datos}

Para resolver los interrogantes anteriormente planteados se emplearon dos métodos: el análisis de redes para estudiar las redes empresariales y la prosopografía, o el estudio de trayectorias y biografías de los actores clave, destapando los vínculos público-privados de las élites. Para el análisis de las redes de las élites empresariales se seleccionaron las 50 mayores empresas de México en 2014 a 
partir del ranking de las 500 corporaciones más importantes de América Latina (América Economía, 2015), y se recolectó información sobre los miembros de los consejos de administración y los grandes accionistas (con más de un 5\% de las acciones) utilizando los informes anuales de las empresas. Para el análisis de redes de interlocking directorates se identificaron aquellos consejeros (directivos) que pertenecían a varios consejos de administración. Para el análisis de los enlaces de propiedad se identificaron aquellos accionistas que eran propietarios en más de una de las 50 empresas. Mediante el uso del software UCINET 6, se construyeron matrices relacionales y se mapeo la red de interlocking directorates, así como la red de enlaces de propiedad.

El uso de la prosopografía aporta información acerca de la composición social y los tipos de relaciones entre las élites, las normas sociales y las prácticas culturales, identificando cómo logran "transmitir [y legitimar] la manera de ser y de ver el mundo" (Gerard y Wagner, 2015). Los estudios de caso fueron seleccionados en función de aquellos actores que tuvieron tanto puestos de importancia en el gobierno de C. Salinas, como un vínculo con el sector privado a partir del año 2000. Los datos empleados fueron obtenidos de informes anuales de las grandes empresas, diccionarios biográficos y boletines.

En este trabajo se estima que es impracticable realizar un análisis estadístico cuantitativo sobre el uso de puertas giratorias de las élites mexicanas. Esto se debe al insuficiente número de casos y a la inaccesibilidad de la información, por eso nos enfocamos en un análisis de casos representativo mediante el método cualitativo, único camino que puede enriquecer el debate, mostrando un cierto patrón de comportamiento dentro de las élites mexicanas.

\section{Resultados}

\section{Redes de interlocking directorates}

La mayoría de las 50 mayores empresas en México están conectadas entre sí por compartir miembros del consejo de administración. La Figura 1 muestra la red empresarial (también denominada red corporativa), donde los nodos representan las empresas y las líneas representan los directivos compartidos. Se empleó el análisis de redes -específicamente los indicadores de cohesión de la red-para conocer el grado de unión de las empresas mexicanas debido a sus élites empresariales. Para comprender comparativamente el nivel de cohesión de la red empresarial de México, se comparó con investigaciones previas que utilizaron una muestra similar (Cárdenas, 2012). La tabla 1 muestra que los valores más altos de cohesión en diversos países del mundo son los de la red corporativa mexicana. 
Figura 1. Red empresarial en México por interlocking directorates

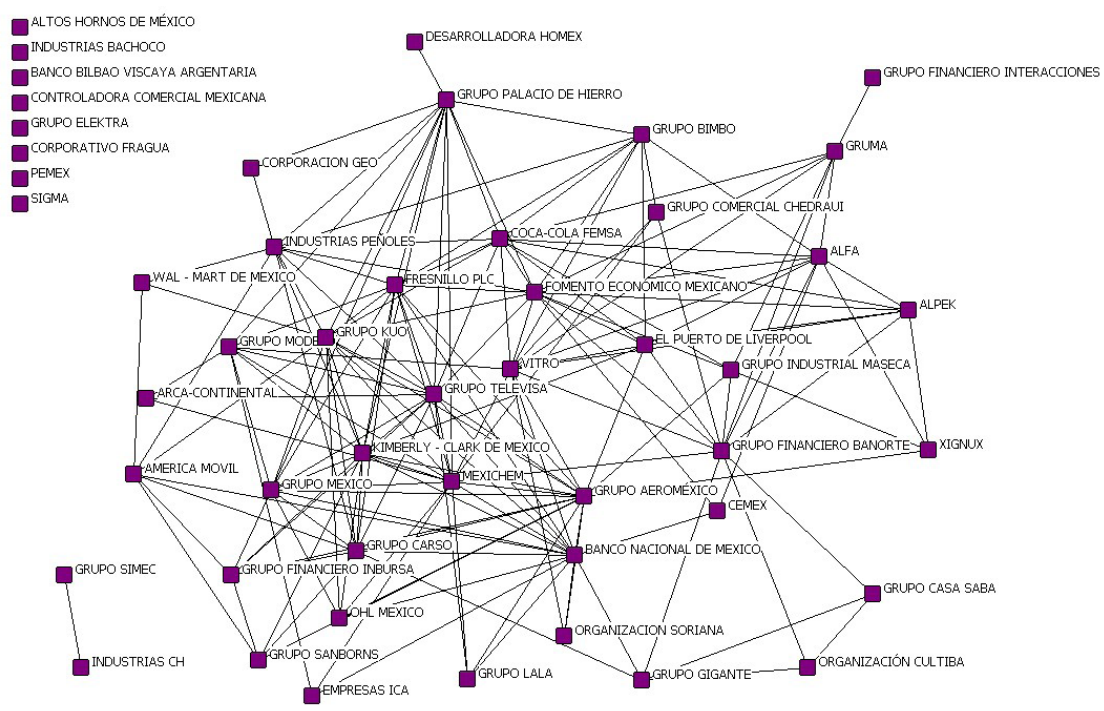

Fuente: Elaboración propia. Nodos: empresas; líneas: directivos compartidos.

Tabla 1. Comparación de los indicadores de cohesión de la red

\begin{tabular}{lccccc}
\hline & Densidad & Grado medio & Compacidad & $\begin{array}{c}\text { Aislados } \\
(\%)\end{array}$ & Tamaño \\
\hline México & 0,14 & 6,76 & 0,36 & 16 & 50 \\
\hline Italia & 0,14 & 6,72 & 0,42 & 5 & 50 \\
Francia & 0,11 & 5,40 & 0,36 & 4 & 50 \\
Canadá & 0,11 & 5,24 & 0,39 & 6 & 50 \\
España & 0,10 & 4,96 & 0,40 & 4 & 50 \\
Alemania & 0,10 & 4,96 & 0,35 & 10 & 50 \\
Suecia & 0,08 & 4,04 & 0,27 & 16 & 50 \\
Países Bajos & 0,08 & 3,84 & 0,27 & 22 & 50 \\
Australia & 0,08 & 3,80 & 0,35 & 8 & 50 \\
EEUU & 0,07 & 3,48 & 0,32 & 10 & 50 \\
Suiza & 0,04 & 2,16 & 0,16 & 28 & 50 \\
Reino Unido & 0,03 & 1,48 & 0,12 & 32 & 50 \\
Japón & 0,02 & 1,12 & 0,04 & 42 & 50 \\
\hline
\end{tabular}

Fuente: Elaboración propia a partir de Cárdenas (2016a) y Cárdenas (2014).

Densidad: número de enlaces existentes dividido entre el total de enlaces posibles. 
Grado medio: promedio de relaciones (suma de enlaces) sobre el total de nodos (tamaño de la red). Compacidad: cohesión basada en la distancia, es decir, probabilidad de cohesión completa en un solo paso en función de las distancias más cortas entre dos pares de nodos.

Aislados: nodos sin ningún enlace.

Tamaño: número de nodos en la red, con y sin enlaces.

Indicadores calculados con matriz dicotomizada.

Las élites empresariales en México forman una red muy cohesionada, es decir, las empresas de México comparten ampliamente directivos entre sus consejos de administración. Esta cohesión no se produce solo en el interior del grupo empresarial, sino entre empresas de diferentes grupos económicos o conglomerados. Las direcciones de las grandes corporaciones de México están abiertas no solo a miembros de la familia propietaria, sino a otras familias. Además, llama la atención que esta cohesión nacional de las élites empresariales en México se produce más allá de las ciudades donde se ubica la sede central de las empresas. Hay relaciones directivas (interlocking directorates) entre empresas asentadas en la Ciudad de México y en Monterrey.

Estudios previos muestran que esta cohesión dentro de las élites empresariales en México es histórica. Musacchio y Read (2007) mostraron que a inicios del siglo XX las élites empresariales estaban mucho más integradas que las élites corporativas brasileñas. Salas-Porras (2006) evidenció un cierto deterioro de la fortaleza de las relaciones empresariales entre 1981 y 2001, pero el presente análisis muestra que las élites empresariales están altamente cohesionadas internamente.

\section{Redes entre propietarios de las grandes empresas}

Las redes entre los propietarios de las grandes empresas mexicanas son analizadas mediante la existencia de enlaces de propiedad. Las propietarios o grandes accionistas están conectados si tienen acciones en las mismas empresas, es decir, si comparten el control de alguna de las grandes empresas. De las 50 empresas seleccionadas, se identificó a aquellos propietarios con más del 5\% del capital accionarial de la empresa. Posteriormente se indagó qué propietarios comparten acciones en alguna de las 50 grandes empresas. Con esta información se construyó la red de propietarios de las grandes empresas mexicanas. Los nodos representan los propietarios y las líneas indican las empresas en las que comparten propiedad (Figura 2). De esta forma se examina el grado de cohesión entre los grandes accionistas de las empresas. 


\section{Figura 2. Red de propietarios de las grandes empresas en México}

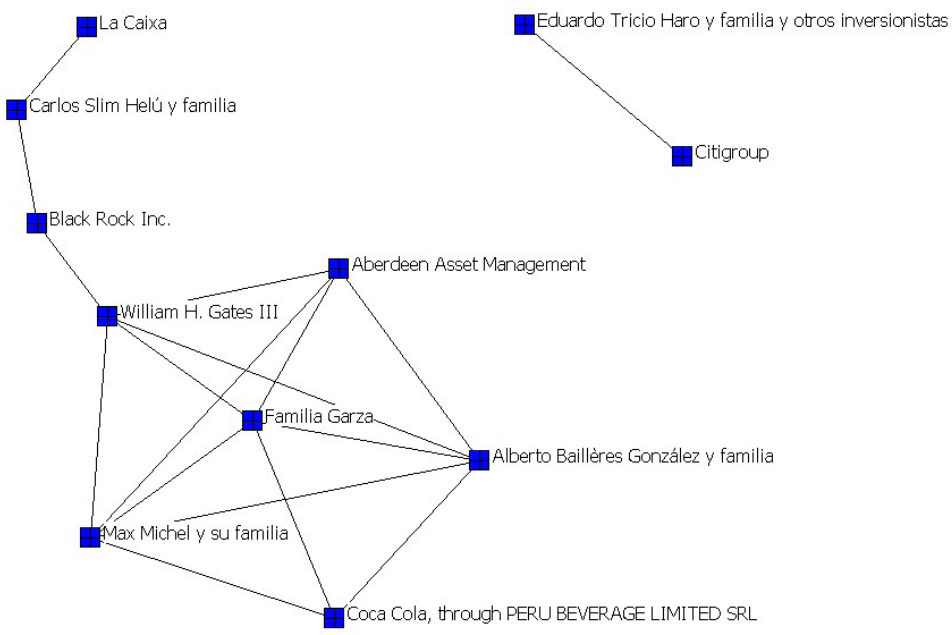

Fuente: Elaboración propia. Nodos: propietarios más del 5\% del capital accionarial; líneas: enlaces de propiedad por ser propietarios de la misma gran empresa.

El análisis de la red de propietarios de las grandes empresas en México revela que son pocos los propietarios que comparten empresas entre sí. La red de enlaces de propiedad es mucho menos cohesionada que la red de interlocking directorates, como se aprecia en la Figura 2. Solo 11 propietarios invierten conjuntamente en varias de las grandes empresas. De estos 11 propietarios múltiples, 6 son empresas o individuos de origen extranjero, lo que revela que se produce conexión entre la clase propietaria mexicana solo gracias a los inversionistas extranjeros. Este hallazgo indica que la cohesión de las élites empresariales en México se produce a través de los consejos de administración pero no mediante la propiedad de las empresas. El control de la propiedad parece seguir en manos de grandes familias mexicanas y solo abierta a grandes inversores extranjeros como Blackrock, La Caixa, Citigroup, o Aberdeen Asset Management.

Puertas giratorias (pantouflage)

En la siguiente sección se analiza una serie de casos de movimientos de servidores públicos al sector empresarial, y viceversa, ocurridos a partir del año 2000 para observar las redes político-empresariales. Nuestra investigación establece un muestreo para los estudios de caso alrededor de las principales secretarías de Estado: Secretaría de Hacienda y Crédito Público (SHCP), Secretaría de Comunicaciones y Transportes (SCT), Secretaría de Relaciones Exteriores (SRE), Secretaría de Energía (SE) y la paraestatal Petróleos Mexicanos (PEMEX), lo cual revela los 
medios donde se inicia la socialización, en un contexto de privatizaciones de gran envergadura (1988-1994). Nos enfocamos en aquellas personas que tuvieron, $a$ posteriori, algún desempeño en el sector privado después del año 2000 (aunque en ocasiones también el regreso a puestos públicos). De esta forma se observan ciertos patrones de relación y comportamiento.

\section{El cliqué Pedro Aspe, Luis Videgaray, J. A. Meade, Peña Nieto}

Una de las redes clave fue construida alrededor de Pedro Aspe Armella, y su cliqué (Golob, 1997). Cuando P. Aspe ocupó la SHCP bajo las órdenes de C. Salinas (1988-1994), tenía como consejero al joven Luis Videgaray (1992-1994). Dos años después de dejar la función pública (1996), P. Aspe fundó la consultora privada Protego para dar servicios de equilibrio en finanzas a gobiernos estatales y secretarías de Estado. Desde el año 2001, el director de finanzas públicas de Protego será Luis Videgaray, el cual fue elegido a partir de 2005 como Secretario de Finanzas durante la gubernatura del Estado de México de Enrique Peña Nieto (2005-2011). Posteriormente, cuando Peña obtiene la presidencia (2012-2018), Luis Videgaray será nombrado tanto Ministro de la SHCP (2012-2016) como Canciller de la SRE (2016-2018). Intercambiará los puestos con José A. Meade, quien dirigió la SRE (2012-2015), coordinó la Secretaría de Desarrollo Social (SEDESOL) y finalmente fue nombrado Ministro de la SHCP (2016-2018), puesto al que renunció para postularse como candidato presidencial.

Una década después de su fundación, Protego se fusiona con Evencore Partners (2006), relación que concluye en 2016, dando paso a una nueva empresa llamada Glisco Partners Inc. (Glisco, s.f.). Esta última hace parte, de acuerdo con los periodistas de ICIJ, de las empresas que los Paradise Papers sacaron a la luz denunciando cuentas offshore en la isla de Barbados (ICIJ, 2016). Después del escándalo de los Paradise Papers, J. A. Meade, jefe de la SHCP, declaró que el gobierno investigaría, pero que no existía punto de ilegalidad en tener capitales en las islas (El Financiero, 2017). En efecto, Meade se codeó en las aulas del ITAM (Instituto Tecnológico Autónomo de México, propiedad de Alberto Baillères) con Videgaray, antiguo director de Protego (propiedad de Aspe). Durante la presidencia de Peña Nieto, Meade y Videgaray compartieron puestos de alta jerarquía en el gabinete.

Vemos por lo tanto la formación de un cliqué, grupo cohesionado de cuatro actores que ocuparon puestos de elevada importancia, se vincularon secretarios y presidente (Videgaray, Aspe, Meade y Peña Nieto), secretarías (SHCP y SRE), consultora (Protego), un gobierno estatal y la presidencia de la República.

\section{Luis Téllez}

Otra de las redes se estableció alrededor de Luis Téllez Kuenzler, quien fue tesorero de la SHCP (1988-1990), asistente del Ministerio de Agricultura (19911994), jefe de la oficina presidencial (1994-1997) y Ministro de la SE (19972000) con el PRI como partido gobernante. Posteriormente, deja el sector público para colaborar (2003-2006) con la firma estadounidense privada de capitales de 
inversión Carlyle Group (Camp, 2010: 186 y 2011: 942; Hernández Rodríguez, 2011: 92-93; Rodríguez, 2017). Inmediatamente después, en 2006, vuelve al sector público como Ministro de la SCT, puesto ocupado hasta 2009, con el PAN como partido gobernante. En 2006, fue también nombrado board director de la consultora Sempra Energy del sector energético, con negocios en EE.UU., México y Sudamérica. Sempra Energy tiene su sede San Diego (California), y es parte de las 500 empresas más importantes de servicios energéticos, con un ingreso de 11,7 mil millones de dólares en 2005 y de 8 mil millones en 2009 (Sempra, 2006, 2010).

En ese año (2009), L. Téllez deja la SCT y se convierte en director del Grupo Bolsa Mexicana de Valores (BMV) desde 2009 hasta 2014, puesto que conservó indistintamente bajo la presidencia del PAN y del PRI. Al renunciar en 2014, bajo la presidencia de Peña Nieto, Téllez fue nombrado "miembro independiente" del comité técnico del Fondo Mexicano del Petróleo, FMP (FMP, 2015), como parte de la llamada Reforma constitucional en materia de energía. Este fondo se encarga de la estabilización y desarrollo del sector petrolero y depende directamente de la SHCP, bajo el gobierno del PRI. No obstante, un año después de dejar la BMV, Luis Téllez será también nombrado senior advisor de las oficinas en México de $K K R \& C o$, fondo de inversión internacional con sede en Nueva York, líder en inversión de proyectos energéticos (Bloomberg KKR, s.f.).

Mientras Luis Téllez se desempeñaba "miembro independiente" de la FMP, en 2015, dirige al equipo local de la consultora Fundación Everis, la cual tiene una ganancia de 1,2 billones de euros, cuenta con 21 mil especialistas y es dirigida por Eduardo Serra Rexach, Secretario de Defensa de los ex-presidentes españoles Felipe González (1984-1988) y José M. Aznar (1996-2000) (Everis, s.f.). Al mismo tiempo, Luis Téllez es consejero de múltiples empresas, la minera Grupo México, la embotelladora-cervecera FEMSA (heredera de la fortuna de los Garza Sada), de la consultora McLarty, y del banco español BBVA Bancomer, entre otras.

Vemos en conclusión que Luis Téllez establece vínculos en ocasiones al mismo tiempo con sectores públicos y privados, indistintamente bajo gobiernos de partidos políticos diferentes, PRI y PAN, y los gobiernos de Salinas (1988-1994), Zedillo (1994-2000), Calderón (2006-2012) y Peña Nieto (2012-2018). Tuvo vínculos con empresas de fondos de inversión globales (KKR \& Cia y Carlyle), consultoras del sector energético (Everis, Sempra Energy, McLarty), secretarías de Estado (SCT, Secretaría de Energía y SHCP), un organismo público inaugurado por Peña Nieto (FMP), empresas exitosas del país (Grupo México y FEMSA), un banco (BBVA) y la Bolsa Mexicana de Valores (Figura 3). Esto nos lleva a observar que dentro de los sectores energéticos, y sobre todo petroleros, hay actores que fluctúan fácilmente entre ambos sectores, desde el público hacia el privado. 


\section{Figura 3: Red egocéntrica de Luis Téllez}

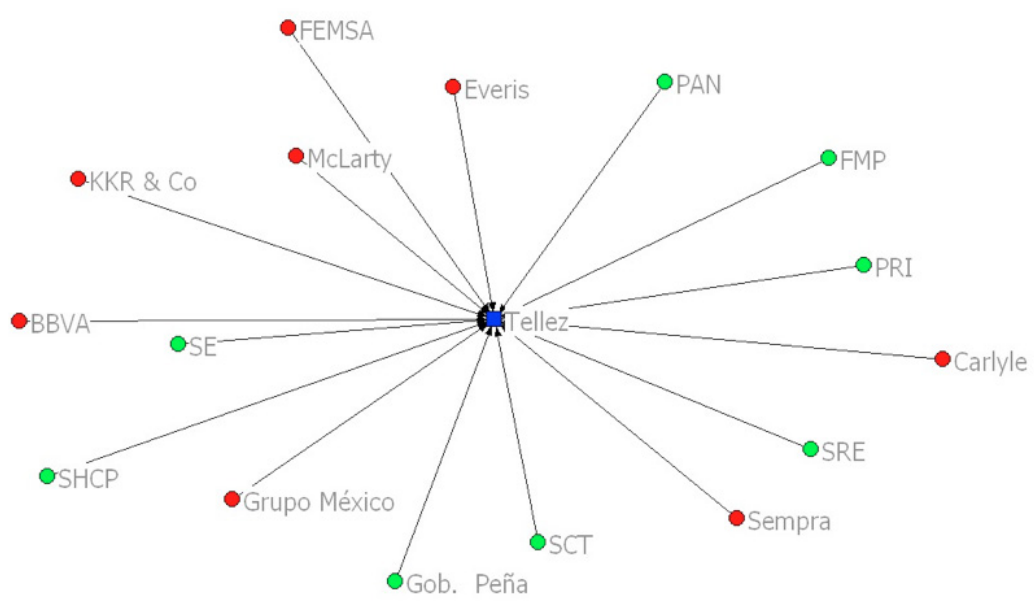

Fuente: Elaboración propia a partir de Camp (2011). Las líneas indican cualquier tipo de colaboración, desde consejo de administración hasta director general o secretario. El color verde muestra el sector público y el color rojo el privado.

\section{Los Subsecretarios}

Por otro lado, algunos actores durante la presidencia de C. Salinas tuvieron puestos de subsecretarios (SS), ya sea vinculados a la SHCP (bajo las órdenes de P. Aspe) o a la SRE, cuando el PRI controlaba la presidencia. El primero fue F. Gil Díaz uno de los actores más trascendentales, puesto que, después de su colaboración con Aspe (y el PRI), se vinculó con la empresa de comunicaciones Avantel en la década de1990 para enseguida regresar al más elevado rango de la función pública en el ámbito financiero en tanto que secretario de la SHCP (2000-2006), bajo el gobierno del PAN. Luego de 2006, vuelve al sector de comunicaciones como Director General de la empresa española Telefónica México. Además, es consejero independiente del BBVA y de la BMV (Banxico, s.f.), esta última dirigida por Téllez. En la Tabla 2 se observan los puestos de los Subsecretarios.

Tabla 2. Subsecretarios

\begin{tabular}{l|l|l|l|l}
\hline \multirow{2}{*}{ Funcionario } & \multicolumn{2}{|l|}{ Puesto 1988-2000 } & \multicolumn{2}{l}{ Puesto desde 2000 } \\
\cline { 2 - 5 } & Público & Privado & Público & Privado \\
\hline \multirow{2}{*}{ F. Gil Díaz } & SS SHCP & \multirow{2}{*}{ Avantel } & S-SHCP & Telefónica \\
& SS-SHCP, SCT, & & & Sempra y Grupo \\
C. Ruiz S.* & PEMEX & & & México \\
P. Cerisola & S. Aeroméxico & Telmex & S-SCT & Indra
\end{tabular}


E. Berruga C. SER Grupo Modelo
A. Ocaranza
C. SER
Vitro y Walmart
G. Borrego S. IMSS
FEMSA
J. Rebolledo
C. SER
Grupo México y
SCC

Fuente: Elaboración propia a partir de Camp (2011). S: Secretarios; SS: Subsecretarios; C: Consejeros y las empresas con las que colaboraron desde el año 2000. Incluimos Aeroméxico y el IMSS (Instituto Mexicano de Seguro Social) porque también son designados por el presidente en turno.

*C. Ruiz será analizado en la sección "La red de PEMEX" debido a que fue director de la paraestatal.

Otros subsecretarios de Aspe también ocuparon puestos tanto públicos como privados. Por mencionar algunos ejemplos, Juan Rebolledo Gout fue consejero de la presidencia (1992-1994), y secretario privado de C. Salinas. Trabajó como asistente de relaciones bilaterales (1994-1997) y de América del Norte y Europa (1997-2000) en colaboración con la SRE. Empero, en 2001 fue nombrado vicepresidente del Grupo México, y en 2003, director de Southern Copper Corp (SCC), la cual es subsidiaria del Grupo México. En su paso por SCC coincidió con C. Ruiz Sacristán.

Otro ejemplo es Enrique Berruga Filloy, quien inicia su carrera política como consejero del expresidente M. de la Madrid (1982-1988), para posteriormente convertirse en responsable de prensa del gobierno en Washington y Londres (1987-1990), de donde fue enviado a Dublín como Embajador de México en Irlanda (1991-1992), para enseguida convertirse en secretario privado de la Secretaría de Relaciones Exteriores (SRE) los últimos años del salinato (1993-1994). En 1997 fue nombrado Embajador en Costa Rica hasta el cambio de milenio, cuando se volvió a incorporar a la SRE bajo las órdenes del excanciller Jorge Castañeda (2000-2003). El último puesto público que ocupó fue de Embajador de México en la ONU (2003-2007), puesto al que renuncia después de casi 25 años como servidor público para convertirse en vicepresidente del Grupo Modelo (2007), una de las empresas cerveceras más importantes del país y recientemente comprada por la multinacional belga-estadounidense Anheuser-Busch InBev con sede en Lovaina, Bélgica. El ex-diplomático y ex-vicepresidente de Grupo México, y articulista del periódico El Universal, omite su colaboración con el sector privado en su biografía, y solo menciona que fue co-presidente para la reforma del sistema mundial del medio ambiente en la Asamblea General de las Naciones Unidas (Opinión El Universal, s.f.).

Otro actor importante, menos visible pero con una sorprendente conversión del sector público hacia el privado, es Antonio Ocaranza. En tanto que funcionario colaboró con la SRE, reemplazando a Berruga como representante diplomático en Washington, fue el encargado de firmar el gran acuerdo de libre comercio con los EEUU, TLCAN (1990-1993), para enseguida convertirse en representante del 
gobierno mexicano en Canadá (1993-1994). Los años siguientes estará vinculado con la oficina de información internacional del gobierno (1995-1998). A finales del siglo XX, dejó el sector público para incorporarse como consultor a Public Strategies Mexico Inc, (PSM, 1998-2002), y dirigir una de las empresas más importantes del país, la vidriera Vitro (2002-2006) y después en director de comunicación de la empresa Walmart, líder mundial en la gran distribución.

Finalmente, un discreto actor es P. Cerisola, el cual inicia su carrera en una paraestatal, Aeroméxico (1990), puesto que dejó para incorporarse el año siguiente a Teléfonos de México, paraestatal privatizada por C. Salinas y comprada por Carlos Slim en 1993, donde estuvo hasta 1999, cuando asumió la dirección de la SCT. En 2007, funda la consultora Factibilis y, a partir de 2016, será el director de la filial mexicana del Grupo Indra (2017), empresa con sede oficial en Madrid, especializada en consultoría y tecnología de negocios en todo el mundo, la cual obtuvo 3.011 millones de euros en ventas, cuenta con 40.000 profesionales, tiene presencia en 140 países, y adquirió 202 millones de euros en inversión (Indra, 2019).

\section{La red de PEMEX}

Un análisis de los antiguos directores de la empresa paraestatal más importante del país, Petróleos Mexicanos (PEMEX), revela que es un sector en el cual las puertas giratorias son medianamente recurrentes. La Tabla 3 muestra que algunos directores de PEMEX (Ruiz, Lajous, Montemayor, Ramírez, Reyes, Suárez), tuvieron vínculos con empresas privadas del sector energético (petrolero y minero) bajo las presidencias del PRI o del PAN, indistintamente.

Tabla 3. Directores de la paraestatal PEMEX (Petróleos Mexicanos)

\begin{tabular}{|c|c|c|}
\hline Nombres & $\begin{array}{l}\text { Directores } \\
\text { (Público) }\end{array}$ & Empresas en sector energético (Privado) \\
\hline C. Ruiz Sacristán & 1994 & $\begin{array}{l}\text { SCC (Grupo México); Sempra (2007-2012); IEN } \\
(2012-2019)\end{array}$ \\
\hline A. Lajous V. & 1994-1999 & $\begin{array}{l}\text { McKinsey \& Co (2001-2011); Schlumberger } \\
\text { (2002-2014); Ternium y Técnicas reunidas }\end{array}$ \\
\hline J. Reyes Heroles & 2006-2009 & Morgan Stanley (2012) \\
\hline L. Ramírez & 2004-2006 & Oro Negro (2012) \\
\hline J. Suárez Coppel & 2009-2012 & $\begin{array}{l}\text { Jacobs Engineering Group; Grupo Modelo; } \\
\text { Televisa }\end{array}$ \\
\hline
\end{tabular}

Fuente: Elaboración propia a partir de Camp (2011); Rodríguez (2017); Pérez (2016, 2010); Bloomberg (s.f); Grupo Modelo (s.f.); IENova (s.f.); Técnicas reunidas (s.f.); Sempra (2010). 
Tres de estos directores (Ruiz, Lajous y Suárez) tuvieron amplios vínculos con multinacionales energéticas (Escamilla, 2016; Carriles, 2014). Ruiz Sacristán no solo dirigió PEMEX (1994), sino que posteriormente ocupó la SCT (1994-2000), puesto ocupado también por otros actores analizados en el presente trabajo, como P. Cerisola (2000-2006) y L. Téllez (2006-2009). Ruiz Sacristán fue nombrado chief executive officer en Infraestructura Energética Nova, IENova, puesto que conservó hasta 2018, cuando es ascendido a executive chairman (IENova, s.f.). IENova es "una empresa de Sempra Energy" con más de 900 empleados y \$7,6 mil millones de dólares invertidos (Bloomberg IEnova, s.f.), la misma donde Luis Téllez se desempeñó como miembro del consejo de administración (board directors). C. Ruiz Sacristán fue también director independiente de Southers Copper Corp (subsidiaria del Grupo México, segunda empresa más importante del país), donde trabajó Rebolledo Gout. Por otro lado, la familia de Ruiz Sacristán también ocupa posiciones clave, como el hermano Jaime Ruiz, quien sustituye a Luis Téllez en la dirección de la Bolsa Mexicana de Valores en 2015 (McLarty, s.f.).

Adrián Lajous Vargas tuvo también un paso importante en el sector público, dirigiendo la paraestatal PEMEX durante cinco años (1994-1999). Lajous se vinculó como senior energy advisor de McKinsey \& Company (2001-2011), firma de consultoría de gestión energética global con presencia en 120 ciudades, 14.000 consultantes y 90 años de experiencia (McKinsey Locations, s.f.). Posteriormente, A. Lajous V. trabajó en Técnicas reunidas (Técnicas reunidas, s.f.) y su hijo, Tomás Lajous Loaeza será el encargado dentro de McKinsey del sector energético mexicano en las oficinas neoyorquinas de la consultora (McKinsey Locations, s.f.).

Igualmente, otro director de PEMEX fue Suárez Coppel (2009-2012), quien, después de este alto puesto en la función pública al mando de la paraestatal mexicana más importante, inició su colaboración con Jacob Engineering Group, siendo al mismo tiempo consejero de Grupo Modelo y de la cadena televisiva Televisa.

A modo de síntesis, la Figura 4 muestra los principales nodos analizados en la presente investigación, revela tanto la centralidad de los puestos gubernamentales (color verde) como los lugares de interacción donde coinciden en múltiples ocasiones los actores analizados (color azul). Las líneas son ilustrativas del vínculo que muestran los actores con el sector público y el privado (color rojo). Estas líneas pueden significar tanto haber tenido un puesto de director general o secretario de Estado, como ser solamente miembro del consejo de administración de una empresa, con lo cual se pretende revelar la pluralidad de los vínculos establecidos y no la importancia del puesto. Muestra la versatilidad de puestos públicos de algunos actores (Téllez, Gil, Meade, Videgaray) o lo menos variable de otros (Lajous, Cerisola, Berruga, Ramírez, Ocaranza). Esto puede significar patrones de comportamiento paralelos, los primeros en sectores inversores, de consultoría o telecomunicaciones; los segundos, en el sector energético y en las principales empresas del país. 
Figura 4: La red de actores analizados

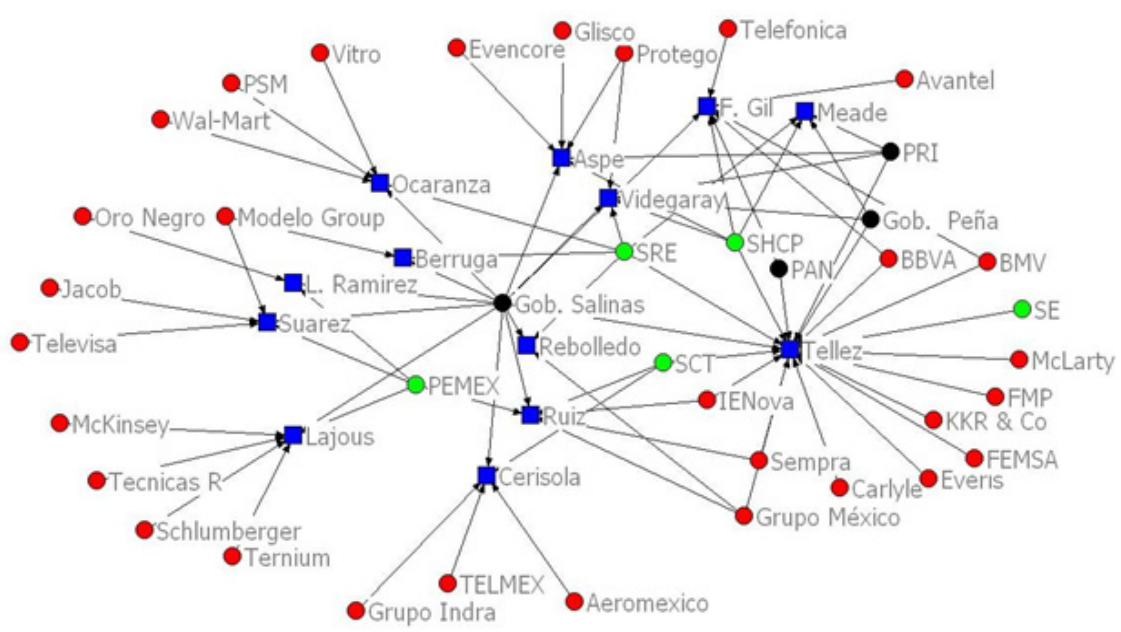

Fuente: Elaboración propia a partir de Camp (2011) y Pérez (2010, 2016). Nodos verde: organismos de Estado (Secretarías, paraestatales); rojo: sector privado; negro: partidos políticos; azul: actores.

¿Son las grandes empresas en la red corporativa de interlocking directorates las que también tienen puertas giratorias?

Podemos observar vasos comunicantes entre el análisis de las redes interlocking directorates de las 50 empresas más importantes de México y el trabajo de identificación de puertas giratorias. Encontramos al menos trece empresas que aparecen conectadas en la red corporativa de directivos compartidos, y que además tienen conexiones con las élites políticas a través de movimientos de empresarios al sector público (Tabla 4). De esta manera, podemos sugerir que dentro del restringido grupo analizado de actores (13), dichos sujetos tuvieron acceso al 26\% de las empresas más importantes del país (13/50), con sus distintas filiales y conglomerados. Es posible observar en la Tabla 4 los nombres de las empresas, la cantidad de empresas con las que están conectadas vía interlocking directorates (compartir directores) y aquellas empresas o grupos económicos donde las élites políticas estudiadas usaron las puertas giratorias. 
Tabla 4. El vínculo entre la red corporativa de interlocking directorates y puertas giratorias

\begin{tabular}{|c|c|c|}
\hline $\begin{array}{l}\text { Empresa en la muestra de } \\
\text { las } 50 \text { más grandes del país }\end{array}$ & $\begin{array}{l}\text { Número de empresas con las } \\
\text { que conecta por compartir } \\
\text { directivos }\end{array}$ & $\begin{array}{l}\text { Empresa o grupo } \\
\text { económico al que } \\
\text { pertenece la empresa } \\
\text { con casos de puerta } \\
\text { giratoria }\end{array}$ \\
\hline Grupo Aeroméxico & 17 & Aeroméxico \\
\hline Banco Nacional de México & 16 & Grupo México \\
\hline Grupo Televisa & 16 & Televisa \\
\hline $\begin{array}{l}\text { Fomento Económico } \\
\text { Mexicano }\end{array}$ & 15 & FEMSA \\
\hline Vitro & 14 & Vitro \\
\hline Coca Cola FEMSA & 13 & FEMSA \\
\hline Grupo México & 12 & Grupo México \\
\hline Grupo Modelo & 9 & Grupo Modelo \\
\hline América Móvil & 7 & TELMEX \\
\hline Grupo Sanborns & 5 & TELMEX \\
\hline Walmart México & 3 & Walmart \\
\hline PEMEX & 0 & PEMEX \\
\hline BBVA & 0 & BBVA \\
\hline
\end{tabular}

Fuente: Elaboración propia a partir de la información citada en el presente texto.

Los resultados muestran que las empresas o grupos económicos con puertas giratorias son empresas bien conectadas en la red corporativa. Por ejemplo, el grupo Televisa, cuyo propietario principal es Emilio Azcárraga y donde también tienen participación accionarial el fondo de inversión Blackrock y "Bill" Gates, tiene conexión con otras 16 grandes empresas a través de sus directivos compartidos (interlocking directorates) como Alberto Baillarès (miembro del consejo de administración de Grupo Bal, Industrias Peñoles, Palacio del Hierro y Fomento Económico Mexicano, entre otros), o Eduardo Tricio Haro (miembro del consejo de administración de Grupo Lala, Mexichem, Aeroméxico, y vicepresidente de la asociación empresarial Consejo Mexicano de Negocios). Además, un antiguo directivo de Televisa, J. Suárez Coppel, quien fuera tesorero corporativo entre 1995 y 1997, fue posteriormente director general la paraestatal PEMEX. Por tanto, la conexión entre una empresa y el Estado es también el puente que tiene el resto de las empresas de la red para acceder al Estado. Es decir, el acceso directo que tienen 
algunas empresas al sector político genera un acceso indirecto al Estado para muchas otras grandes empresas debido a la alta conexión de las élites empresariales entre sí.

\section{Discusión y conclusiones}

Las élites empresariales en México son de las más cohesionadas de América Latina (Cárdenas, 2016a). La cohesión de las élites empresariales se sustenta en redes de interlocking directorates, que permiten organizar los diversos intereses sectoriales, al tiempo que facilita la negociación con el Estado, como observamos a través del uso de puertas giratorias por una parte de las élites. Además, el control de las asociaciones empresariales por parte de las élites empresariales, las cuales son las principales financiadoras y las que dirigen estas organizaciones, conduce a que las políticas formuladas vayan encaminadas a defender sus intereses particulares, por encima de los generales de todo el sector. A pesar de los vínculos entre los principales grupos económicos, la propiedad de las empresas mexicanas sigue estando altamente concentrada y no abierta a cualquier inversor, en especial si es un gran propietario nacional. Futuros estudios deberían profundizar qué está generando la llegada de inversionistas extranjeros a las empresas mexicanas. ¿Es el inicio de un cambio en el gobierno corporativo dominado por los grupos económicos familiares? ¿Supondrá la modificación del tipo de capitalismo imperante en México?

Nuestra investigación revela que las empresas que han tenido puertas giratorias son empresas bien conectadas en la red corporativa. Ello sugiere que las puertas giratorias son una vía de conexión política de casi todas las élites empresariales, no solo de algunas. Las empresas o grupo económicos conectados con el Estado hacen de puente entre el resto de las élites empresariales y las élites políticas. Por lo que se intuye que las demandas políticas de las élites empresariales son demandas de clase o élite, y no solo de grupos económicos particulares. Solo unos cuantos grupos económicos tienen vínculo directo con el Estado por las puertas giratorias, pero todas las élites empresariales se pueden beneficiar debido a las altas conexiones que tienen entre de ellos. Tal vez la cohesión de las élites empresariales las hace más fuertes para acceder al Estado, y el propio acceso al Estado refuerza aún más la necesidad de cohesionarse. En otros países de América Latina se ha constatado la amplia extensión de las puertas giratorias, como en Argentina (Castellani, 2018) y Chile (Maillet, González-Bustamante y Olivares, 2019), por lo que sería conveniente seguir investigando acerca de la relación entre cohesión empresarial y puertas giratorias, especialmente cuando emergen los debates en torno a la necesidad de regular las prácticas de paso de ex-políticos al sector privado.

Los trabajos de captura del Estado en América Latina sobre las tramas de Odebrecht (Durand, 2018) y la concentración de riqueza en un mínimo grupo de personas y empresas (Cañete Alonso, 2018) forman parte de una nueva veta de investigación que muestra la alta concomitancia de las élites empresariales y políticas. Algunos autores proponen que, si bien se ha fraguado una "élite de la globalización" fuertemente vinculada con los EEUU (Luna Ledesma y Velasco, 2012), nuestra investigación contribuye al análisis de la composición social y 
los vínculos nacionales de una parte de las élites, específicamente aquella que vivió, por un lado, la apertura democrática electoral y, por otro, la incorporación de sectores empresariales al campo político. Nuestro análisis de los miembros del gabinete de C. Salinas revela la existencia amplia de puertas giratorias en México, pero queda por conocer si esta ha sido la pauta dominante en todos los gobiernos. Futuros trabajos deberían incluir información sobre funcionarios públicos desde 1988 hasta 2018, momento en el que se produce una ruptura del sistema de partidos con la llegada de Andrés Manuel López Obrador a la presidencia (Aragón Falomir, Fernández y Lucca, 2019). ¿Se mantienen las puertas giratorias en un gobierno de izquierdas? ¿Cuál es el origen del gabinete del presidente de México Andrés Manuel López Obrador? ¿Supone una ruptura con las élites empresariales dominantes, o bien se trata de una continuidad?

Nuestros resultados mostraron que tanto las élites empresariales mexicanas como las élites políticas que incursionan en el sector privado están extremadamente cohesionadas. En el caso de las primeras, esto se da gracias a redes de interlocking directorates; en las segundas, debido a sus trayectorias compartidas. Concluimos por lo tanto que existen excelsas redes vinculantes entre las empresas mexicanas, que no solo comparten directores, sino que en ocasiones estos directores son reclutados desde el sector público, lo que genera un vínculo del sector privado con un miembro de un gobierno o partido político. Estos hallazgos proponen la emergencia de una clase dominante público-privada, y reportan la posible capacidad de las élites empresariales de capturar al Estado.

Nuestra investigación ilustra también la importancia curricular de pasar por puestos públicos para escalar posteriormente a cargos privados. Para ello, describimos hacia qué lugares y qué actores establecieron un movimiento transversal, enganchándose durante ciertos períodos al sector público y durante otros al privado. Los resultados muestran una conflagración tanto de redes empresariales, como de élites empresariales y de élites políticas, que pareciera propiciar el movimiento entre sectores. Estos desplazamientos entre sectores públicos y privados muestran una anomalía en los controles o restricciones constitucionales, o posibles conflictos de interés, principalmente con aquellos actores que colaboraron en la misma área económica, tanto en el sector público como en el privado.

El uso de análisis de redes en el estudio de las élites posibilita mapear la consabida - pero a veces poco evidenciada- concomitancia entre poder económico y política. Además, permitió ejemplificar el comportamiento corporativo e individual, facilitando así la visualización tanto de las principales empresas del país como de los actores políticos que han tenido puestos de alta importancia en el sector público. Los nodos construidos en torno a actores individuales ilustraron que los sectores energéticos (petroleros y mineros) y de telecomunicaciones son las principales ventanas de oportunidad para que antiguos funcionarios públicos "giren".

Además, en la presente investigación se muestra que esta clase dominante público-privada está sustentada en una red empresarial cohesionada, donde los intereses particulares de cada empresa tienden a estar coordinados y asociados antes que enfrentados o en álgida competencia. Aunque solo un grupo de empresarios pasa 
al sector político, las políticas defendidas tienden a ser en beneficio del conjunto de élites empresariales, y no solo de un grupo económico específico. Ello sugiere que la captura del Estado puede estar facilitada por una cohesión de una súperélite, aunque ello requiere análisis comparativos futuros, especialmente entre economías que evidencian una alta captura del Estado, como Panamá, y economías con una menor captura, como Uruguay.

Este trabajo se enmarca también en el proceso de transformación global de reestructuración de las relaciones y de marcos regulatorios de acción individual después de las reformas neoliberales. Es en este momento en que los entramados de trayectorias oscilan titilantes entre puestos públicos y directivos de empresas. Observamos que los actores podían colaborar indistintamente con los dos principales partidos, lo cual habla también de un pragmatismo que ha fraguado una inédita clase público-privada tan cohesionada como las élites empresariales analizadas, y con una nueva capacidad a transformarse, diseminarse y sobrevivir.

Demostramos así que las élites existen, interactúan entre sí y están fuertemente cohesionadas, tanto desde el punto de vista individual como corporativo. Es verosímil afirmar que hay vasos comunicantes para que coordinen y tengan acciones colectivas. La agenda a futuro es continuar destapando sus estructuras latentes de interacción, y construyendo minuciosas redes que den cuenta de la complejidad de sus movimientos para entender por qué se sigue reproduciendo una organización social endogámica y cohesionada.

\section{Bibliografía}

Aguayo, S. (2007). Vuelta en U. México D. F., México: Taurus.

Aitken, R. (2008). Carlos Salinas de Gortari. En W. Fowler, Gobernantes mexicanos: 1911-2000, México D. F., México: Fondo de Cultura Económica.

Alba Vega, C. (1996). Los empresarios y el Estado durante el salinismo. Foro Internacional, 36 (143/144), 31-79.

Alba Vega, C. (2006). Los empresarios y la democracia en México. Foro Internacional, 46 (183), 122-149.

América Economía (2015). Las 500 mayores empresas de América Latina. Disponible en: http:// rankings.americaeconomia.com/2013/ranking_500_latinoamerica_2013/. Última consulta: 25/12/2015.

Aragón Falomir, J.; Fernández, A. y Lucca, J. (2019). Las elecciones de 2018 en México y el triunfo del Movimiento de Regeneración Nacional (Morena). Estudios Políticos (Universidad de Antioquia), 54, 286-308.

Babb, S. (2003). Proyecto México: los economistas del nacionalismo al neoliberalismo. México D. F., México: Fondo de Cultura Económica.

Banxico (s.f.). Disponible en: http://www.anterior.banxico.org.mx/publicaciones-y-discursos/ publicaciones/seminarios/banco-de-mexico_-independencia-de-los-bancos-centr/\%7B1321ADF3684C-37BC-067C-6DDA32888FF0\%7D.pdf. Última consulta: 15/01/2019.

Bloomberg Infraestructura energética N (s.f). Disponible en: https://www.bloomberg.com/research/ stocks/people/person.asp?personId=9067622\&privcapId=225078297. Última consulta: 15/01/2019.

Bloomberg KKR \& Co (s.f.). Disponible en: https://www.bloomberg.com/research/stocks/people/ person. asp?personId=684639\&privcapId=2140. Última consulta: 15/01/2019.

Boltanski, L. (2009). De la critique: Précis de sociologie de l'émancipation. Paris, Francia: Gallimard. Camp, R. A. (1995). Political Recruitment Across Two Centuries. Mexico 1884-1991. Austin, Estados 
Unidos: University of Texas Press.

Camp, R. A. (2002). Mexico's Mandarins: Crafting a Power Elite for the 21st Century. Berkeley, Estados Unidos: University of California Press.

Camp, R. A. (2010). The Metamorphosis of Leadership in a Democratic Mexico. New York, Estados Unidos: Oxford University Press.

Camp, R. A. (2011). Mexican Political Biographies, 1935-2009. Austin, Estados Unidos: University of Texas Press.

Camp, R. A. (2012). The Oxford Handbook of Mexican Politics. New York, Estados Unidos: Oxford University Press.

Cañete Alonso, R. (2018). Democracias capturadas: el gobierno de unos pocos. Oxfam Internacional, Clacso. Disponible en: https://oxfamilibrary.openrepository.com/bitstream/handle/10546/620600/rrcaptured-democracies-161118-es.pdf?sequence=1\&isAllowed=y

Cárdenas, J. (2012). Varieties of Corporate Networks: Network Analysis and fsQCA. International Journal of Comparative Sociology, 53(4), 298-322.

Cárdenas, J. (2014). El Poder Económico Mundial: Análisis de redes de interlocking directorates y variedades de capitalismo. Madrid, España: CIS.

Cárdenas J. (2016a). Enredando a las élites empresariales en América Latina: análisis de redes de interlocking directorates y propiedad en México, Chile, Perú y Brasil. América Latina Hoy, 73, 15-44. Cárdenas, J. (2016b). Why Do Corporate Elites Form Cohesive Networks in Some Countries and Do Not in Others? Cross-National Analysis of Corporate Elite Networks in Latin America. International Sociology, 31(3), 341-363.

Cárdenas, J. y Robles-Rivera, F. (2018) Business elite networks and state capture in Central American countries. Working paper. Disponible en: https://papers.ssrn.com/sol3/papers.cfm?abstract_ id $=2896153$

Carriles, L. Ex funcionarios, listos para capitalizar apertura. El Economista (2014, 6 de enero). Disponible en: https://www.eleconomista.com.mx/empresas/Ex-funcionarios-listos-para-capi talizarapertura-20140107-0121.html

Castellani, A. (2018). Lobbies y puertas giratorias. Los riesgos de la captura de la decisión pública. Nueva Sociedad, 276, 48-61.

Dezalay, Y. y Garth, B. (2002). La Mondialisation des guerres de palais. Paris, Francia: Seuil.

Domínguez, J. (Ed.) (1997). Technopols: Freeing Politics and Markets in Latin America in the 1990s. Pennsylvania, Estados Unidos: Penn State University Press.

Durand, F. (2016). Cuando el poder extractivo captura el Estado. Lobbies, puertas giratorias y paquetero ambiental en Perú. Lima, Perú: OXFAM.

Durand, F. (2018). Odebrecht, la empresa que capturaba gobiernos. Lima, Perú: Fondo Editorial PUCP.

Grupo Modelo. Enrique Berruga, es nombrado Vicepresidente de Relaciones corporativas y Comunicación de Grupo Modelo (2007, 22 de agosto). Disponible en: http://www.latibex.com/act/ esp/empresas/hechosrelev/2007/833F1hecho20070823_1301.pdf

El Financiero. No es ilegal tener dinero en el extranjero: Meade sobre 'Paradise Papers' (2017, 6 noviembre). Disponible en: https://www.elfinanciero.com.mx/economia/no-es-ilegal-tener-dinero-enel-extranjero-meade-sobre-paradise-papers

Escamilla O. Los 6 exdirectores de PEMEX que hicieron ganar a multinacionales. Poder (2016, 24 de mayo). Disponible en: https://www.rindecuentas.org/reportajes/2016/05/24 /exdirectores-pemex-quehicieron-ganar-multinacionales/

Everis Board Directors (s.f.). Disponible en: https://www.everis.com/mexico/es/about-us/boarddirectors. Última consulta: 15/01/2019.

Ferrari, M. (2010). Prosopografía e historia política Algunas aproximaciones. Antitesis, 5, 529-550.

Fondo Mexicano del Petróleo (2015). Informe Anual 2015. Disponible en: https://www.fmped.org.mx/ transparencia/\%7B1E8DCEDE-0118-7A34-640C-2911D5D8277C\%7D.pdf

Fuchs, D. A. (2007). Business Power in Global Governance. Boulder, Estados Unidos: Lynne Rienner Publishers, Inc. 
Gérard, E. y Wagner, A-C. (Dirs.) (2015). Élites et savoirs. Cahiers de la recherche sur l'éducation et les savoirs, 14.

Glisco Partners Inc Team (s.f.). Disponible en: http://gliscopartners.com/en/index. Última consulta: $15 / 01 / 2019$.

Golob, S. (1997). Pedro Aspe and the Next Mexican "Miracle". En J. Domínguez, Technopols. Pennsylvania, Estados Unidos: Penn State University Press.

Hernández Rodríguez, R. (1998). Presidencialismo y élite en el cambio político de México. Política $y$ Gobierno, 5 (1).

Hernández Rodríguez, R. y Pansters, W. (2012). "La democracia en México y el retorno del PRI”. Foro Internacional, 52 (4).

ICIJ (2016). Barbados DataBase - Paradise Papers Secrets of the global elite. Disponible en: https:// offshoreleaks.icij.org/nodes/ 101740574

Ienova Quienes somos (s.f.). Disponible en: http://www.ienova.com.mx/quienes_somos.php. Última consulta: $15 / 01 / 2019$.

Indra. Indra refuerza su apuesta por México con una nueva sede corporativa en Querétaro. Comunicado de prensa (2017, 14 de julio). Disponible en: https:/www.indracompany.com/sites/default/ files/170714_np_indra_inaugura_oficinas_en_queretaro.pdf

INDRA. Entre las empresas líderes en innovación de su sector en Europa por inversión y creación de valor. Comunicado de prensa (2019, 19 diciembre). Disponible en: https://www.indracompany.com/ sites/default/files/191219_np_indra_lider_en_innovacion_en_europa.pdf

Labastida, M. y Camou, A. (2001). Globalización, identidad y democracia: México y América latina. México D. F., México: Siglo XXI.

Loaeza, S. (2010). Acción Nacional. El apetito y las responsabilidades del triunfo. México, D. F., México: El Colegio de México.

Luna Ledesma, M. y Velasco, J. (2012). El TLCAN, la Comisión trilateral y la "conexión” mexicana: entre la eficacia y la legitimidad. En M. Luna Ledesma y A. Salas-Porras, ¿Quién gobierna América del norte? Élites, redes y organizaciones (pp.149-196). México, D. F., México: SITESA.

Maillet, A., González-Bustamante, B. y Olivares, A. (2019). Public-Private Circulation and the Revolving door in the Chilean Executive Branch (200-2014). Latin American Business Review, 20 (4), 367-387.

McLarty (s.f.). Luis Téllez. (2019, 19 diciembre). Disponible en: https://maglobal.com/team/luistellez/. Última consulta: 15/01/2019.

McKinsey Locations (s.f.). Disponible en: https://www.mckinsey.com/locations. Última consulta: $15 / 01 / 2019$.

Mendieta, J. y Schmidt, S. (2005). Estudios sobre la red politica de México. México, D. F., México: UNAM.

Mills, W. (1956). The Power Elite. New York, Estados Unidos: Oxford University Press.

Mizruchi, M. (2016). The Fracturing of the American Corporate Elite. Cambridge, Estados Unidos: Harvard University Press.

Musacchio, A. y Read, I. (2007). Bankers, Industrialists, and Their Cliques: Elite Networks in Mexico and Brazil During Early Industrialization. Enterprise and Society, 8 (4), 842-880.

Opinión (s.f.). Articulistas. El Universal. Disponible en: http://www.eluniversal.com.mx/autoropinion/articulistas/enrique-berruga-filloy. Última consulta: 15/08/2019.

Ortiz Gallegos, J. (2011). La Mancha azul. Del PAN al NeoPAN y al PRIoPAN. México, D. F., México: Grijalbo.

Pérez, A. (2010). Camisas azules, manos negras: el saqueo de Pemex desde los Pinos. México, D. F., México: Gandhi.

Pérez, A. (2016). PEMEX RIP: Vida y asesinato de la principal empresa mexicana. México, D. F., México: Grijalbo.

Ramírez, P. Las consentidas de PEMEX (y de Lajous). Reporte Indigo. (2013, 1 de octubre). Recuperado de: https://www.reporteindigo.com/reporte/las-consentidas-de-pemex-y-de-lajous/

Rodríguez, I. Posible conflicto de interés de Téllez Kuenzler en el FMP. Periódico La Jornada. (2017, 
16 de junio). Disponible en: https://www.jornada.com.mx/2017/06/16/economia/021n1eco

Rodríguez, J. (2003). Revisitando el poder: cambios en la estructura social del poder en España (19912000). Revista Sistema, 172.

Rodríguez, J., Cárdenas, J. y Oltra, C. (2006). Redes de poder económico en Europa. Revista Sistema, 194, 3-44.

Rouban, L. (2010). L'inspection générale des Finances, 1958-2008: pantouflage et renouveau des stratégies élitaires. Sociologies pratiques, 2 (21).

Rousseau, I. (1999). Mexique: Une révolution silencieuse? Élites gouvernementales et projet de Modernisation. Paris, Francia: L'Harmattan.

Salas-Porras, A. (2006). Fuerzas centrípetas y centrífugas en la red corporativa mexicana (1981-2001). Revista Mexicana de Sociología, 68 (2), 331-375.

Salas-Porras, A. y Luna Ledesma, M. (2012). ¿Quién gobierna América del norte? Élites, redes y organizaciones. Ciudad de México: SITESA.

Scott, J. (2008). Modes of Power and the Re-Conceptualization of Elites. The Sociological Review, 56, 25-43. Disponible en: https://doi.org/10.1111/j.1467-954X.2008.00760.x

Sempra energy names Luis Téllez to corporate board of directors (2006, 9 de junio). Disponible en: https://www.sempra.com/newsroom/press-releases/sempra-energy-names-luis-tellez-corporate-boarddirectors. Última consulta: 15/01/2019.

Sempra energy names Luis Téllez to corporate board of directors (2010, 28 de septiembre). Disponible en: https://www.sempra.com/newsroom/press-releases/sempra-energy-names-luis-tellez-corporateboard-directors-0. Última consulta: 15/01/2019.

Serna, M. (2018). Les patrons latino-américains prennent le pouvoir. Le Monde diplomatique.

Serna, M. y Bottinelli, E. (2018). El poder fáctico de las élites empresariales en la política latinoamericana: un estudio de ocho países. Buenos Aires, Argentina: CLACSO.

Silva, E. (1996). The State and Capital in Chile: Business Elites, Technocrats, and Market Economics. Boulder, Estados Unidos: Westview Press.

Smith, P. H. (1976). Labyrinths of Power: Political Recruitment in Twentieth-Century Mexico. Princeton, Estados Unidos: Princeton University Press.

Técnicas Reunidas CV ALV (2018). Disponible en:: http://www.tecnicasreunidas.es/wp-content/ uploads/2018/11/CV_ALV_EN.pdf. Última consulta: 15/01/2019.

Teichman, J. A. (2001). The Politics of Freeing Markets in Latin America: Chile, Argentina and Mexico. Londres, Inglaterra: The University of North Carolina Press.

Tirado, R. (2012). Las élites de América del Norte en la génesis del TLC. En A. Salas Porras y M. Luna Ledesma, ¿Quién gobierna América del Norte? Élites, redes y organizaciones. México D. F., México: SITESA.

Tirado, R. y Luna Ledesma, M. (1986). La politización de los empresarios mexicanos (1970- 1982). En J. Labastida, Grupos económicos y organizaciones empresariales en México. México, D. F., México: Alianza Editorial Mexicana-UNAM.

Useem, M. (1984). The Inner Circle. New York, Estados Unidos: Oxford University Press.

Weyland, K. (1998). The fragmentation of business in Brazil. En F. Durand y E. Silva. (Eds.), Organized Business, Economic Change, and Democracy in Latin America. New Brunswick, Estados Unidos: Transaction Press.

Woldenberg, J. (2012). Historia mínima de la transición democrática en México. México, D. F., México: El Colegio de México.

Zepeda Patterson, J. (2007). Los Amos de México. México, D. F., México: Planeta.

Jaime Aragón Falomir y Julián Cárdenas, “Análisis de redes empresariales y puertas giratorias en México: Cartografía de una clase dominante público-privada". Revista Temas y Debates. ISSN 16660714, año 24, número 39, enero-junio 2020, pp. 81-103. 